\section{Setting up of a liquid chromatography-high resolution tandem mass spectrometry method for the detection of caseins in food. A comparison with ELISA method}

\section{Daniela Gastaldi, ${ }^{1}$ Claudio Baiocchi, ${ }^{1}$ Valeria Giancotti, ${ }^{2}$ Elisabetta Genta, ${ }^{3}$ Lucia Decastelli ${ }^{4}$}

'Dipartimento di Chimica, Università di Torino; ${ }^{2}$ Marini Group, Moncalieri; ${ }^{3}$ Marini Group Laboratorio, Rivoli; ${ }^{4}$ Istituto Zooprofilattico Sperimentale del Piemonte, Liguria e Valle d'Aosta, Controllo Alimenti e Igiene delle Produzioni, Torino, Italy

\section{Abstract}

Determination of caseins in food matrices is usually performed by using the competitiveenzyme-linked immunosorbent assay (ELISA) technique. However such a technique suffers from a number of limitations. Among these, the applicability to a narrow concentration range, a non linear (logarithmic) response, a non-negligible cross-reactivity and a high cost per kit. At the time of the completion of this study, in case of ELISA positive feedback, there was poor availability in the literature of finding reliable instrumental methods able to determine both qualitatively and quantitatively this class of substances. In the present study, a liquid chromatography-high resolution tandem mass spectrometry (HPLC-HRMS/MS) instrumental method was developed with a high resolution mass spectrometer (Orbitrap). Real samples of sausages in which caseins were detected by ELISA technique were analysed. A casein-free sample of ham was used as a blank. The analytical characteristics of the instrumental method were compared with those of a commercial ELISA test, declared specific for $\alpha$ - and $\beta$-casein.

\section{Introduzione}

L'allergia al latte è una delle allergie alimentari più comuni nei bambini a partire dalla nascita fino a 1 anno di età. Fortunatamente, l'85\% dei bambini si desensibilizza entro i primi 3 anni di vita. Non esiste alcun trattamento per l'allergia, e l'unico modo per controllare i sintomi è evitare l'assunzione degli allergeni. Il latte intero contiene circa il 3\% di proteine, l' $80 \%$ delle quali è rappresentato dalle caseine, le quali sono considerate i principali allergeni presenti nel latte (Natale, 2004), anche se è stato dimostrato che la maggior parte delle proteine del latte, ancorché presenti a basse concentrazioni, sono potenziali allergeni (Wal, 2004).

Per la determinazione della caseina in matrici alimentari, si utilizza attualmente la tecnica competitive-enzyme-linked immunosorbent assay (ELISA), la quale però soffre di una serie di limitazioni. Tra queste l'applicabilità in un intervallo di concentrazioni piuttosto ristretto (1,5-4,5 ppm in soluzione), la risposta non lineare (logaritmica) caratterizzata quindi da maggiore incertezza, un costo pro kit elevato, e una non trascurabile cross-reactivity (Rozenfeld, 2002). Proprio per quest'ultimo motivo, in caso di riscontro ELISA positivo, sono necessarie analisi di conferma. Attualmente, in letteratura c'è scarsa disponibilità di metodi strumentali affidabili di determinazione qualitativa e quantitativa di questa classe di sostanze.

Nell'ultimo decennio, la spettrometria di massa è diventata il principale strumento per l'identificazione di peptidi e proteine. Il campione viene digerito con una proteasi, solitamente tripsina, e i peptidi prodotti vengono separati in high-performance liquid chromatography (HPLC) e analizzati in tandem mass spectrometry (MS/MS). Lidentificazione della sequenza amminoacidica dei diversi frammenti proteolitici viene effettuata mediante un algoritmo di ricerca in un database, sulla base dei relativi spettri MS/MS. La sequenza amminoacidica di appropriati peptidi identifica in modo univoco la proteina di cui sono parte.

In questo lavoro è stato sviluppato un metodo strumentale liquid chromatography-high resolution tandem mass spectrometry (HPLCHRMS/MS) con uno spettrometro di massa ad alta risoluzione (Orbitrap) per la determinazione di caseine in matrici carnee. La metodica è stata messa a punto su campioni di insaccati in cui la presenza di caseina era dichiarata in etichetta. Un campione di prosciutto dichiarato esente da caseina è stato utilizzato come blank, ovvero come matrice per la costruzione delle rette di calibrazione e per il calcolo della resa di estrazione. Le prestazioni analitiche del metodo strumentale sono state confrontate con quelle di un test ELISA commerciale, dichiarato specifico per $\alpha$ - and $\beta$ caseina.

\section{Materiali e Metodi}

Circa un grammo di campione di insaccato, finemente sminuzzato, è stato posto in una provetta con tappo a vite, e omogeneizzato con Ultra-Turrax in presenza di $10 \mathrm{~mL}$ di tampone
Correspondence: Valeria Giancotti, Marini Group, via Vittime del Vajont 18, 10024 Moncalieri, Italy. Tel. +39.0119592817 - Fax: +39.0119592818 .

E-mail: vgiancotti@marinigroup.it

Key words: Allergens, Caseins, Liquid chromatography, High resolution mass spectrometry, ELISA test.

Received for publication: 15 January 2013. Revision received: 30 April 2013. Accepted for publication: 1 May 2013.

This work is licensed under a Creative Commons Attribution 3.0 License (by-nc 3.0).

(c) Copyright D. Gastaldi et al., 2013

Licensee PAGEPress, Italy

Italian Journal of Food Safety 2013; 2:e27

doi:10.4081/ijfs.2013.e27

TRIS/glicina $(0,1 \mathrm{M} / 0,4 \mathrm{M})$ a $\mathrm{pH}$ 8.7. Dopo riscaldamento sotto agitazione a $45^{\circ} \mathrm{C}$ per $2 \mathrm{~h}$, i campioni sono stati raffreddati e ultracentrifugati a $20.000 \mathrm{rpm}$ per $30 \mathrm{~min}$. Aliquote del surnatante, dopo digestione con tripsina, sono state analizzate in HPLC-HRMS/MS

Lo strumento utilizzato è un HPLC Ultimate 3000 (Dionex, Sunnyvale, CA, USA) accoppiato ad un analizzatore di massa ad alta risoluzione (LTQ Orbitrap) con sorgente ionica electrospray (Thermo Scientific, Waltham, MA, USA).

Allo scopo di individuare i peptidi più adeguati per l'analisi quantitativa, $50 \mu \mathrm{L}$ di una soluzione a $10,0 \mathrm{ppm}$ di caseinati di sodio (Sigma-Aldrich, St. Louis, M0, USA) in tampone bicarbonato a pH $8.0(40 \mathrm{mM})$ sono stati miscelati con $50 \mu \mathrm{L}$ di urea e digeriti con tripsina da pancreas bovino (Sigma-Aldrich). I diversi frammenti triptici ottenuti sono stati separati mediante HPLC e rivelati mediante HRMS-MS frammentando gli ioni più abbondanti in ogni spettro. I profili cromatografici degli estratti dei campioni sono stati acquisiti in HRMS/MS, isolando e frammentando soltanto gli ioni di interesse.

Le condizioni analitiche sono le seguenti: colonna RP C18 Phenomenex Synergi (150 $\mathrm{mm} \times 2,0 \mathrm{~mm} \times 3 \mu \mathrm{m}$ ) (Phenomenex Torrance, CA, USA) con precolonna Phenomenex Security Guard $5 \mu \mathrm{m} \times 30 \mathrm{~mm}$ (Phenomenex); flusso $200 \mu \mathrm{L} / \mathrm{min}$; volume di iniezione $20 \mu \mathrm{L}$; temperatura di eluizione: $28^{\circ} \mathrm{C}$; fasi mobili: soluzione acquosa di $\mathrm{HCOOH} \mathrm{0,05 \%} \mathrm{(A)} \mathrm{e} \mathrm{CH}_{3} \mathrm{CN}$ (B). Per l'eluizione è stato effettuato un gradiente lineare dal 95 al $45 \%$ di A in 45 min.

Le condizioni massa sono le seguenti:

Evento 1: Fourier transform mass spectrometry

(FTMS) Res 30.000, range m/z: 450-2000;

Evento 2: FTMS, MS/MS dello ione a $\mathrm{m} / \mathrm{z}$ 742.500 CID 35, Act Q 0,25, Act time 30,00 msec; 
Evento 3: FTMS, MS/MS dello ione a $\mathrm{m} / \mathrm{z}$ 692.900 CID 35, Act Q 0,25, Act time 30,00 msec.

La quantificazione delle caseine è stata eseguita sia mediante calibrazione esterna in matrice sia con il metodo delle aggiunte standard allo scopo di valutare l'esattezza. Prove di recupero sono state effettuate mediante aggiunte standard sul prosciutto dichiarato esente da caseina, dopo averne verificato l'effettiva assenza, alle concentrazioni di 6, 3 e 1,5 $\mu \mathrm{g} / \mathrm{g}$. Per la calibrazione sono state utilizzate 7 soluzioni standard di $\alpha$-caseina (purezza $\geq 99 \%$ ) e $\beta$-caseina (purezza $\leq 97 \%$ ) (SigmaAldrich) nell'intervallo di concentrazione 0-45 $\mu \mathrm{g} / \mathrm{mL}$ per l' $\alpha$-caseina, e $0-75 \mu \mathrm{g} / \mathrm{mL}$ per la $\beta$ caseina.

Per i test ELISA è stato seguito il protocollo fornito dalla ditta produttrice (GenProbe casein kit; Hologic GenProbe Inc., San Diego, CA, USA) utilizzando il tampone estraente incluso nel kit.

Per l'identificazione degli amminoacidi è stata usata la notazione internazionale convenzionale con le lettere riportate in Tabella 1.

\section{Risultati e Discussione}

Dall'analisi del tracciato cromatografico dei frammenti triptici di caseinati di sodio (Figura 1) sono stati identificati due peptidi (uno proveniente dall' $\alpha$ s1-caseina e l'altro dalla $\beta$ caseina), specifici delle caseine bovine (Rif. banca dati Swiss-Prot), con peso molecolare 1383,730 uma (ione a doppia carica: 692,869 uma; sequenza: FFVAPFPEVFGK; dall'amminoacido 38 all'amminoacido 49 dell' $\alpha$ s1-caseina) (Figura 2) e peso molecolare 741,450 uma (ione monocarica; sequenza: GPFPIIV; dall'amminoacido 218 all'amminoacido 224 della $\beta$ caseina) (Figura 3). Le Tabelle 2 e 3 riportano lo schema di frammentazione dei peptidi di interesse.

Il limite di rivelabilità del metodo era di 49,0 ng/mL per l' $\alpha$-casina e di $110 \mathrm{ng} / \mathrm{mL}$ per la $\beta$ caseina. La ripetibilità, calcolata sulla base di 8 determinazioni indipendenti sullo stesso campione (prosciutto esente, positivizzato a 3,0 $\mu \mathrm{g} / \mathrm{g}$ per entrambe le caseine), era del 3,93\% nel caso dell' $\alpha$-caseina e dell' $8,00 \%$ nel caso della $\beta$-caseina. Il recupero era del $70 \pm 5 \%$ per
Tabella 1. Notazione internazionale convenzionale degli amminoacidi.

\begin{tabular}{lcc} 
Amminoacido & Sigla & $\begin{array}{c}\text { Peso } \\
\text { monoisotopico } \\
\text { (uma) }\end{array}$ \\
Glicina & G & 57,0215 \\
Alanina & A & 71,0371 \\
\hline Serina & S & 87,0320 \\
Prolina & P & 97,0528 \\
\hline Valina & V & 99,0684 \\
Treonina & T & 101,0477 \\
\hline Cisteina & C & 103,0092 \\
Isoleucina & I & 113,0841 \\
\hline Leucina & L & 113,0841 \\
Asparagina & N & 114,0429 \\
\hline Acido aspartico & D & 115,0269 \\
Glutammina & Q & 128,0586 \\
\hline Lisina & K & 128,0950 \\
Acido glutammico & E & 129,0426 \\
\hline Metionina & M & 131,0405 \\
Istidina & H & 137,0589 \\
\hline Fenilalanina & F & 147,0684 \\
Arginina & R & 156,1011 \\
\hline Tirosina & Y & 163,0633 \\
Triptofano & W & 186,0793 \\
\hline & &
\end{tabular}

Tabella 2. Frammentazioni del peptide nello spettro di Figura 2 (ione precursore: 1374,7300) che consente di risalire agli amminoacidi costituenti.

\begin{tabular}{|c|c|c|c|c|}
\hline Frammento b* & Ordine successivo di frammentazione ${ }^{\circ}$ & Sigla & Ordine successivo di frammentazione ${ }^{\circ}$ & Frammento $\mathrm{y}^{*}$ \\
\hline 148,0764 & 1 & $\mathrm{~F}$ & 12 & 1384,7300 \\
\hline 295,1441 & 2 & $\mathrm{~F}$ & 11 & 1237,6616 \\
\hline 394,2125 & 3 & V & 10 & 1090,5932 \\
\hline 465,2496 & 4 & A & 9 & 991,5247 \\
\hline 562,3024 & 5 & $P$ & 8 & 920,4876 \\
\hline 709,3708 & 6 & $\mathrm{~F}$ & 7 & 823,4349 \\
\hline 806,4236 & 7 & $P$ & 6 & 676,3665 \\
\hline 935,4662 & 8 & E & 5 & 579,3137 \\
\hline 1034,5346 & 9 & V & 4 & 450,2711 \\
\hline 1181,6030 & 10 & $\mathrm{~F}$ & 3 & 351,2027 \\
\hline 1238,6245 & 11 & $\mathrm{G}$ & 2 & 204,1343 \\
\hline 1366,7195 & 12 & K & 1 & 147,1128 \\
\hline
\end{tabular}

*In riferimento allo schema tipico di frammentazione delle catene peptidiche; ${ }^{\circ}$ calcolato a partire dai lati opposti della catena peptidica.

Tabella 3. Frammentazioni del peptide nello spettro di Figura 3 (ione precursore: 742,4498 ) che consente di risalire agli amminoacidi costituenti.

\begin{tabular}{|c|c|c|c|c|}
\hline Frammento b* & Ordine successivo di frammentazione ${ }^{\circ}$ & Sigla & Ordine successivo di frammentazione ${ }^{\circ}$ & Frammento $\mathrm{y}^{*}$ \\
\hline 58,0287 & 1 & G & 7 & 742,4498 \\
\hline 155,0815 & 2 & $\mathrm{P}$ & 6 & 685,4283 \\
\hline 302,1499 & 3 & $\mathrm{~F}$ & 5 & 588,3756 \\
\hline 399,2027 & 4 & $\mathrm{P}$ & 4 & 441,3071 \\
\hline 512,2867 & 5 & I & 3 & 344,2544 \\
\hline 625,3708 & 6 & I & 2 & 231,1703 \\
\hline 724,4397 & 7 & V & 1 & 118,0863 \\
\hline
\end{tabular}

*In riferimento allo schema tipico di frammentazione delle catene peptidiche; ${ }^{\circ}$ calcolato a partire dai lati opposti della catena peptidica. 


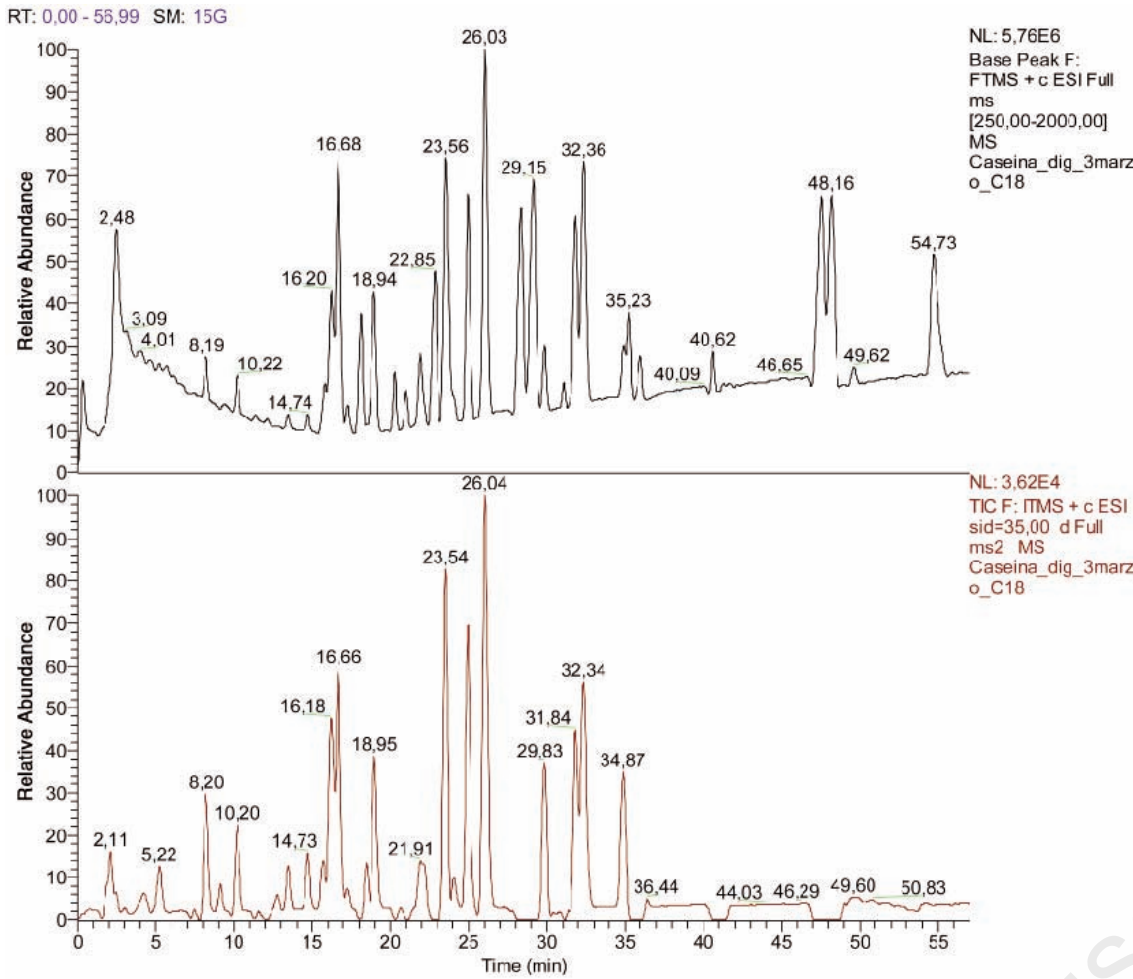

Figura 1. Cromatogramma full mass spectrometry (tracciato nero) e tandem mass spectrometry (tracciato rosso) di un digerito triptico di caseina.

Tabella 4. Concentrazioni di caseina nei campioni analizzati.

\begin{tabular}{lcc} 
Campione & as 1-caseina $(\mathrm{mg} / \mathrm{kg})$ & $\beta$-caseina $(\mathrm{mg} / \mathrm{kg})$ \\
Hamburger bovino & $\mathrm{nr}$ & $\mathrm{nr}$ \\
Hamburger equino & 0,73 & 1,03 \\
\hline Prosciutto C1 & $\mathrm{nr}$ & $\mathrm{nr}$ \\
Salame Cl & 4,47 & 48,1 \\
\hline Salame C2 & $\mathrm{nr}$ & $\mathrm{nr}$ \\
Prosciutto C2 & 7,45 & 25,5 \\
\hline Prosciutto C3 & 27,7 & 10,2 \\
\hline
\end{tabular}

entrambe le caseine. Le concentrazioni di caseina nei campioni reali analizzati sono riportate in Tabella 4.

I risultati indicano una buona ripetibilità del metodo, un ampio intervallo dinamico lineare (oltre 40 ppm) e limiti di rivelabilità e quantificazione dello stesso ordine di quelli del metodo ELISA.

È interessante notare che, nei campioni testati, soprattutto in un campione di salame, la concentrazione di $\beta$-caseina è risultata molto maggiore della concentrazione di $\alpha$ caseina.

Per il confronto dei parametri analitici del metodo strumentale con quelli del metodo ELISA l'estrazione delle caseine è stata effettuata seguendo il protocollo ELISA e utilizzando l'estraente fornito dal kit commerciale; la misura della concentrazione è stata eseguita con entrambe le tecniche analitiche, utilizzando gli stessi calibratori del kit.

In generale, i valori forniti dall'ELISA concordavano molto bene con quelli ottenuti mediante il metodo strumentale per l'as1caseina. Il confronto tra i risultati ottenuti con i due metodi sono riportati in Tabella 5. Come si può evincere dalla tabella, i risultati ottenuti con il metodo della concentrazione esterna, non si discostano in modo significativo dai valori ottenuti con il metodo delle aggiunte standard, indicando una buona accuratezza del metodo utilizzato.

\section{Conclusioni}

I risultati ottenuti hanno messo in evidenza che l'anticorpo del test ELISA da noi utilizzato, sebbene dichiarato specifico per le $\alpha$ - e le $\beta$ caseine, in realtà ha un'affinità maggiore per la $\alpha$-caseina, dovuta a una più favorevole cinetica di reazione. A riprova di questo, diluizioni diverse dell'estratto di salame in cui era presente una maggiore concentrazione di $\beta$ caseina, analizzate con l'ELISA hanno fornito

Tabella 5. Confronto dei risultati ottenuti con i diversi metodi.

\begin{tabular}{|c|c|c|c|}
\hline Sigla campione & $\begin{array}{c}\text { Concentrazione } \\
\text { (HPLC-HRMS/MS) } \\
\text { mediante calibrazione esterna } \\
(\mathrm{mg} / \mathrm{kg})\end{array}$ & $\begin{array}{c}\text { Concentrazione } \\
\text { (HPLC-HRMS/MS) } \\
\text { mediante aggiunte standard } \\
(\mathrm{mg} / \mathrm{kg})\end{array}$ & $\begin{array}{l}\text { Concentrazione } \\
\text { (ELSA) } \\
(\mathrm{mg} / \mathrm{kg})\end{array}$ \\
\hline $\mathrm{Cl}(0 \mathrm{ppm})$ & $\mathrm{nr}$ & $\mathrm{nr}$ & LOD \\
\hline C2 (5,5-7,0 ppm) & 6,50 & 6,13 & 6,55 \\
\hline $\mathrm{SAl}^{*}$ & 5,93 & 5,75 & 5,14 \\
\hline $\mathrm{P}^{*} *$ & 11,1 & 10,3 & 10,6 \\
\hline $\mathrm{HE} 2^{*}$ & 1,66 & 1,02 & 0,54 \\
\hline SA2* & $\mathrm{nr}$ & $\mathrm{nr}$ & $\mathrm{nr}$ \\
\hline
\end{tabular}

HPLC-HRMS/MS, liquid chromatography-high resolution tandem mass spectrometry; ELISA, enzyme-linked immunosorbent assay; Cl, campione di controllo 1 fornito con il kit ELISA; nr, non rinvenuto; LOD, limit of detection; C2, campione di controllo 2 fornito con il kit ELISA; SA1, campione di salame 1; P2, campione di prosciutto 2; HE2, campione di hamburger 2; SA2, campione di salame 2. *Campioni di salami, prosciutto e hamburger equino diversi dai precedenti. 


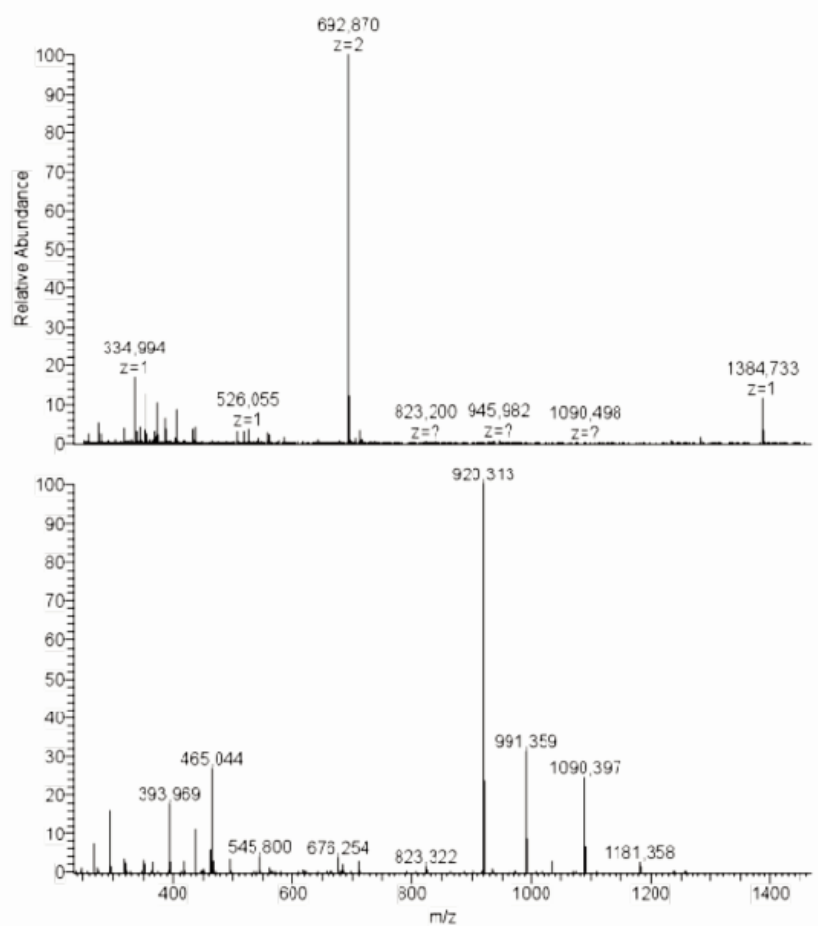

NL: $4,44 E 6$

Caseina_dig_3marzo_C18\#1 + c ESI Full ms

\section{NL. $9,98 \mathrm{E} 3$}

Caseina_dig_3marzo_C18\#16 ESI 25 . ESI Sid=35,00 dFull $[18000-1400$ jo]

Figura 2. Spettro di massa del picco a $\mathbf{2 6 . 0 3}$ min e tandem mass spectrometry dello ione a range $\mathrm{m} / \mathrm{z} 692.87^{+2}$.

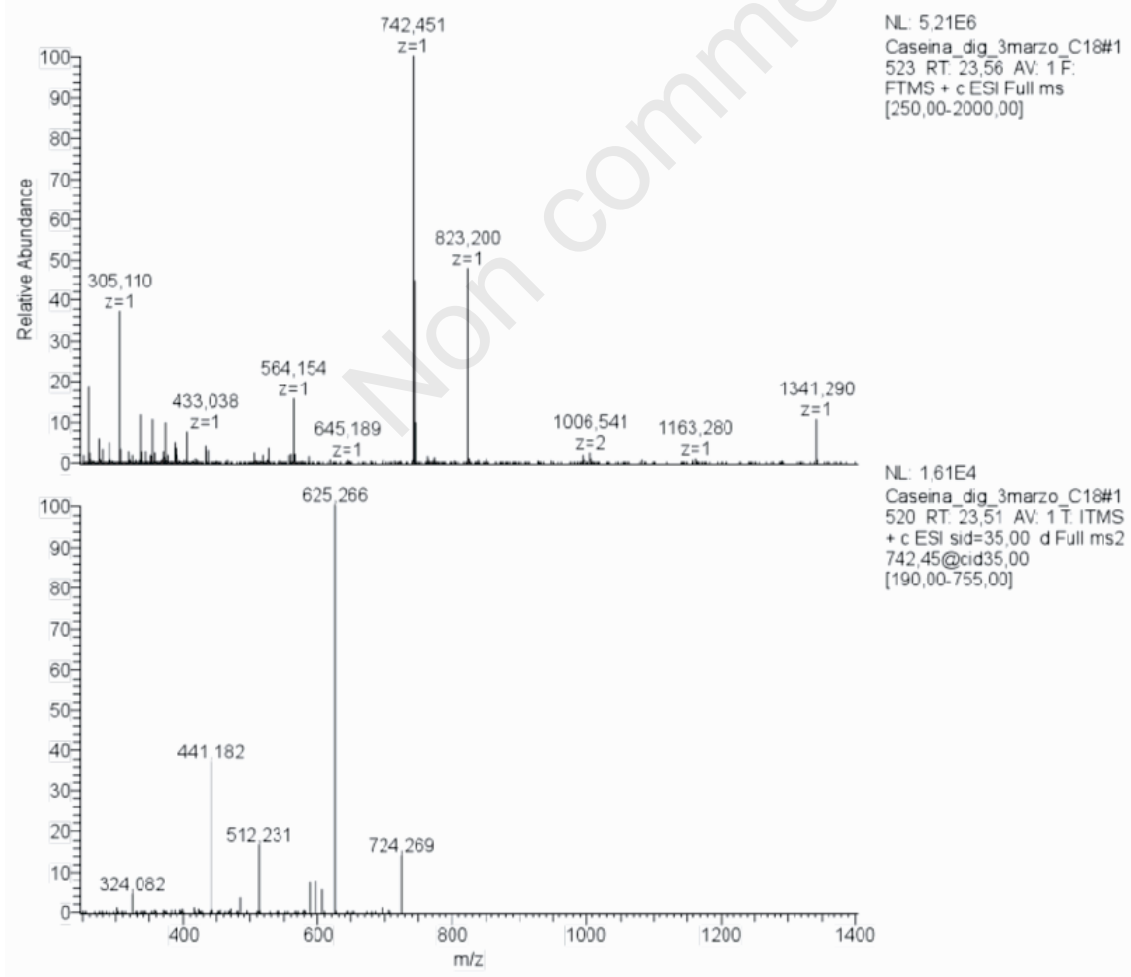

Figura 3. Spettro di massa del picco a $25.56 \mathrm{~min}$ e spettro tandem mass spectrometry dello ione a range $\mathrm{m} / \mathrm{z} 742.45^{+1}$.

valori di concentrazione di caseina sul campione crescenti all'aumentare della diluizione in quanto al diminuire della $\alpha$-caseina diventava cineticamente più competitiva la forma $\beta$.

\section{Bibliografia}

Natale M, Bisson C, Monti G, Peltran A, Perono-Garoffo L, Valentini S, Fabris C, Bertino E, Coscia A, Conti A, 2004. Cow's milk allergens identification by twodimensional immunoblotting and mass spectrometry. Mol Nutr Food Res 48:363-9.

Rozenfeld P, Docena GH, Anon MC, Fossati CA 2002. Detection and identification of a soy protein component that cross-reacts with caseins from cow's milk. Clin Exp Immunol 130:49-58.

Wal JM, 2004. Bovine milk allergenicity. Ann Allerg Asthma Im 93(Suppl.3):s2-s11. 